\title{
EXPRESSION FOR A FUNCTION IN TERMS OF ITS SPHERICAL MEANS
}

\author{
BY H. RHEE
}

Communicated by Avner Friedman, December 1, 1969

Let $f(X)$ be a continuous function in $R^{n}$. The spherical means, $\mathrm{SM}$, of $f$ is defined as follows:

$$
\operatorname{SM}[f ; X, \rho]=\omega_{n}^{-1} \int_{\alpha} f\left(X+\rho_{\alpha}\right) d \omega_{\alpha},
$$

where $X=\left(x, x_{2}, x_{3}, \cdots, x_{n}\right)$ is the center of the sphere of radius $\rho$. $\alpha$ denotes a unit vector. When $\rho=x$, we write $\operatorname{SM}[f ; X, x]=\operatorname{SM} * f$. The main purpose of this paper is to derive an expression for a function $f(X), X \in R_{+}^{n}$ (the open half-space with $x>0$ ), in terms of SM ${ }^{*}$. For $(X, t) \in Q_{+}\left(|t|<x,-\infty<x^{\prime}<\infty, x^{\prime}=\left(x_{2}, x_{3}, \cdots, x_{n}\right),\left(x, x^{\prime}\right)\right.$ $\in R_{+}^{n}, n$ odd $\geqq 3$ ) we define the paraboloidal means, PM, of $f$ as follows:

$$
\operatorname{PM}[f ; X, t]=\omega_{n-1}^{-1}(x+t)^{2-n} \int_{b}^{\infty} d y \int_{\alpha} f\left(y, x^{\prime}+R \alpha\right) R^{n-3} d \omega_{\alpha},
$$

where $b=(x-t) / 2, Y=\left(y, y^{\prime}\right), R=[(x+t)(2 y-x+t)]^{1 / 2}$.

A function $f(X)$ is said to belong to the class $C_{\epsilon}$ in $R_{+}^{n}$, if $f$ is continuous in $R_{+}^{n}$ and $f(X)=O\left(|X|^{(1-n-2 \epsilon) / 2}\right), 0<\epsilon<1$, for large $|X|$. We observe that $\operatorname{PM}[f ; X, t]$ exists, if $f \in C_{\epsilon}$. It is easily verified that if $f \in C_{\epsilon}$, then $\mathrm{SM}^{*} f \in C_{\epsilon}$. The well-known identity on iterated spherical means by John and Asgeiersson [3] states

$$
\int_{\xi} d \omega_{\xi} \int_{\eta} F(r \xi+s \eta) d \omega_{\eta}=2 \omega_{n-1} \int_{|r-s|}^{r+s} J \tau d \tau \int_{\zeta} F(\tau \zeta) d \omega_{\zeta},
$$

where $J=\left[\left((r+s)^{2}-\tau^{2}\right)\left(\tau^{2}-(r-s)^{2}\right)\right]^{(n-3) / 2}(2 r s)^{2-n}$.

Theorem. Let $f \in C_{\epsilon}$ in $R_{+}^{n} \quad(n$ odd $\geqq 3)$, and let $W(X, t)$ $=(x+t)^{n-2} \mathrm{PM}\left[\mathrm{SM}^{*} f ; X, t\right]$. Then the following identity holds for $(X, t) \in Q_{+}$,

(2) $t \mathrm{SM}[f ; X, t]=M_{1} D D_{0}^{n-3} W(X, t)+M_{2} \sum_{i=1}^{(n-3) / 2} a_{i} D_{1}^{i} t^{i+1} \operatorname{SM}[f ; X, t]$,

AMS Subject Classifications. Primary 3506; Secondary 3579.

Key Words and Phrases. Spherical means, paraboloidal means, John-Asgeiersson identity, iterated spherical means, characteristics, Darboux equation. 
where

$$
\begin{aligned}
M_{1} & =(-1)^{(n-1) / 2} \Gamma^{-1}(n-2), D=x \partial / \partial t+t \partial / \partial x, \\
D_{0} & =(x+t)^{-1} D, M_{2}=-\Gamma^{-1}(n-2) 2^{(3 n-11) / 2} \Gamma(k+1), k=(n-3) / 2, \\
a_{i} & =\Gamma(k+i)\left[2^{i-1} \Gamma(i) \Gamma(k-i+2)\right]^{-1}, D_{1}=D(x+t)^{-1} .
\end{aligned}
$$

(The lengthy proof of the theorem which makes use of (1) will be submitted elsewhere.)

From (2) it follows that

$$
f(X)=M_{3} x^{-1}\left[D^{2} D_{0}^{n-3} W(X, t)\right]_{t=0},
$$

where $M_{3}=M_{1}\left[1-M_{2} \sum_{i=1}^{(n-3) / 2} a_{i} \Gamma(i+2)\right]^{-1}$ for $n>3, M_{3}=-1$ for $n=3$. (3) is an expression for $f(X), X \in R_{+}^{n}$, in terms of the paraboloidal means of $\mathrm{SM}^{*} f$.

Lemma. Suppose $f \in C_{\mathbf{c}}$ and $x \quad \partial f / \partial x \in C_{\mathbf{\epsilon}}$. Let $W_{0}[f ; X, t]$ $=(x+t)^{n-2} \mathrm{PM}[f ; X, t]$. Then we have

$$
D W_{0}[f ; X, t]=-W_{0}[\delta f ; X, t] \text {, where } \delta=(\partial / \partial x) x .
$$

Proof. It is easily seen that $D R=y R_{y}, D R^{n-3} f\left(y, x^{\prime}+R \alpha\right)$ $=d / d y\left(y R^{n-3} f\right)-R^{n-3} \partial / \partial y(y f)$. Integrate this expression with respect to $y$ to complete the proof.

Applying (4) to (3) and letting $J(X)=\mathrm{SM} * f$, we obtain

$$
\begin{array}{ll}
f(X)=-(2 \pi x)^{-1} \int_{x / 2}^{\infty} d y \int_{\alpha} \delta^{2} J\left(y, x^{\prime}+R_{0} \alpha\right) d \omega_{\alpha} & \text { for } n=3, \\
f(X)=M_{3} \omega_{n-1}^{-1} x^{2-n} \sum_{i=1}^{n-1} b_{i} \int_{x / 2}^{\infty} d y \int_{\alpha} R_{0}^{n-3} \delta^{i} J\left(y, x^{\prime} R_{0} \alpha\right) d \omega_{\alpha} & \text { for } n>3,
\end{array}
$$

where $R_{0}=[x(2 y-x)]^{1 / 2}$, the constants $b_{i}$ are the coefficients in the expansion of $D D_{1}^{n-3} g(X), g \in C^{n-2}$.

REMARK. It follows from (2) that if we can find $f(X)$ which satisfies the equation $\mathrm{SM}^{*} f=J(X)$ for a given function $J$, then we will have a representation in $Q_{+}$for the even-solutions of the Darboux equation $V_{t t}+(n-1) / t V_{t}-\Delta V=0$ in terms of $J(X)=V(X, \pm x)$ (that is, if the equation $\mathrm{SM}^{*}=J$ can be inverted, then we have an expression for the even-solutions of the Darboux equation in $Q_{+}$in terms of prescribed values on the characteristics $C_{+}(x=t, x>0)$ and $\left.C_{-}(x=-t, x>0)\right)$. The problem of inverting $\mathrm{SM}^{*} f=J(X)$ in $R^{3}$ was studied by Chen [1], [2]. It was shown in [4] that inverting the equation $\operatorname{SM}[f ; X,|X|]=J(X)$ leads to a representation of the evensolutions of the Darboux equation in the exterior of the characteristic 
cones $C_{0}\left(t= \pm|X|, X \in R^{n}, n\right.$ odd $\left.\geqq 3\right)$ in terms of prescribed values on $C_{0}$.

\section{REFERENCES}

1. Y. W. Chen, On the solutions of the wave equation in a quadrant of $R^{4}, \mathrm{Bull}$. Amer. Math. Soc. 70 (1964), 172-177. MR 28 \#4236.

2. - Solutions of a characteristic value problem of the wave equation with regularity conditions at infinity, Mimeographed report, University of Massachusetts, Amherst, Mass.

3. F. John, Plane waves and spherical means applied to partial differential equations, Interscience, New York, 1955. MR 17, 746.

4. H. Rhee, $A$ representation of the solutions of the Darboux equation in odd-dimensional spaces, Trans. Amer. Math. Soc. 150 (1970).

State University College of New York, Oneonta, New York 13820 